\title{
Nordic Walking and the Isa Method for Breast Cancer Survivors: Effects on Upper Limb Circumferences and Total Body Extracellular Water - a Pilot Study
}

\author{
Andrea Di Blasio ${ }^{a}$ Teresa Morano $^{a} \quad$ Giorgio Napolitano $^{a} \quad$ Ines Bucci ${ }^{a} \quad$ Serena Di Santo $^{a}$ \\ Sabina Gallina $^{b} \quad$ Lucia Cugus $^{c}$ Francesco Di Donato $^{d}$ Alberto D'Arielli ${ }^{e}$ Ettore Cianchetti ${ }^{f}$ \\ a Department of Medicine and Aging Sciences, 'G. d'Annunzio' University of Chieti-Pescara, Chieti Scalo, Italy; \\ ${ }^{b}$ Department of Neuroscience and Imaging, 'G. d'Annunzio' University of Chieti-Pescara, Chieti Scalo, Italy; \\ c ‘M. Aresu' Department of Medical Sciences, University of Cagliari, Cagliari, Italy; \\ ${ }^{\mathrm{d}}$ Dietamovimento.it Laboratories, Montesilvano, Italy; \\ e Sportlab, Francavilla al Mare, Italy; \\ ${ }^{f}$ Department of Medical, Oral and Biotechnological Sciences, 'G. d'Annunzio' University of Chieti-Pescara, Chieti Scalo, Italy
}

\section{Keywords}

Lymphoedema - Muscular pump - Physical exercise · Nordic walking $\cdot$ Foam balls

\section{Summary}

Background: The negative side effects of breast cancer treatments can include upper limb lymphoedema. The growing literature indicates that Nordic walking is an effective discipline against several disease symptoms. The aim of this study was to determine whether introduction to Nordic walking alone is effective against total body extracellular water and upper limb circumferences in breast cancer survivors compared to its combination with a series of specifically created exercises (i.e. the Isa method). Methods: 16 breast cancer survivors ( $49.09 \pm 2.24$ years) were recruited and randomly assigned to 1 of 2 different training groups. Results: 10 lessons on Nordic walking technique plus the Isa method significantly reduced both extracellular body water and the extracellular-to-total body water ratio ( $p=0.01$ for both), and also the circumference of the upper limb, (both relaxed arm and forearm circumferences) ( $p=0.01$ for all), whereas Nordic walking alone did not. Conclusions: Introduction to Nordic walking does not seem to affect lymphoedema in breast cancer survivors. This might be because novice Nordic Walkers do not adequately generate an effective muscular pump through coordination of the alternated bimanual openclose cycle. The Isa method appears to close this gap.

(c) 2016 S. Karger GmbH, Freiburg

\section{Introduction}

Both non-pharmacological and pharmacological treatments for breast cancer have negative side effects that can range from the kinesiological to psychological domains, according to the interactions of treatment characteristics with both the physiopathological and psychological conditions of each woman $[1,2]$. Upper limb lymphoedema is a potential negative consequence of breast cancer surgery and radiation therapy, and this can occur during the months after treatment ends, and even years later.

The growing scientific literature underlines the psychophysical importance of engagement in early physical exercise for breast cancer survivors, which can induce an early recovery, along with prevention of breast cancer recurrence and the onset of sedentary lifestyle-related diseases [3]. Focusing our attention here on lymphoedema, guidelines have suggested that early treatment with physiotherapy and practice of physical exercise favours lymphatic drainage [4].

Nordic walking represents a recent physical activity trend, which is a form of brisk walking using walking poles to actively engage the trunk and upper limbs during walking. Due to its executive characteristics, Nordic walking can also be considered as an effective discipline against lymphoedema. Indeed, during Nordic walking, there is an alternated open-close cycle of the hands, which creates a 'pumping effect'; this would theoretically favour both lymphatic and blood circulation through upper limb muscle contraction. However, to maintain the natural gait while the hands are completing the open-close cycle in an alternate manner can be a task that is too complex for people who have not practiced much

\section{KARGER}

() 2016 S. Karger GmbH, Freiburg

Fax +497614520714 
physical exercise previously. Therefore, in this situation, an effective pump effect against lymphoedema can be considered as a delayed effect of Nordic walking, i.e. it occurs only when the correct technique has been learned and is practiced.

The aim of the present study was to determine whether introduction to Nordic walking (i.e. 10 lessons on Nordic walking technique) has any effects on total body extracellular water and upper limbs circumference, compared to the combination of this Nordic walking with a series of exercises specifically created for breast cancer survivors, such as the Isa method.

\section{Materials and Methods}

\section{Subjects}

Sixteen breast cancer survivors (mean age $49.09 \pm 2.24$ years) were recruited by the Department of General Surgery Specialised in Senology of the 'G. Bernabeo' Hospital (Ortona, Italy). The inclusion criteria are reported as online supplementary material. The Ethics Committee of the 'G. d'Annunzio' University of Chieti-Pescara approved this study, and written informed consent was obtained from all participants.

\section{Study Design}

After recruitment, a cardiologist and a sports medicine specialist verified cardiovascular and orthopaedic eligibility for Nordic walking of participants. After reaching medical eligibility (T0), the participants underwent anthropometry and body composition analysis before beginning the learning phase of the training for Nordic walking (i.e. 10 lessons on the technique). After these baseline tests, the participants were randomly assigned to 1 of the 2 different train-

Fig. 1. Isa balls applied to Nordic walking poles.

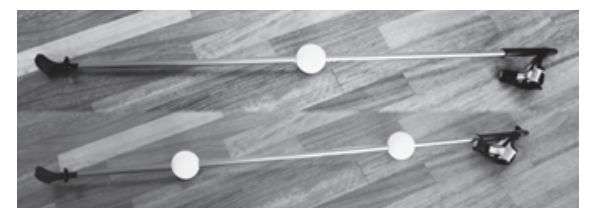

ing groups: the Nordic walking alone group (NW group, $\mathrm{n}=8$ ) or the Nordic walking plus the Isa method group (NW-Isa group, $\mathrm{n}=8$ ). The anthropometry and body composition analyses were repeated at the end of this learning phase (T1).

Anthropometry and extracellular water assessment methods are reported in the online supplementary material.

\section{Isa Method}

The Isa method includes a series of dynamic exercises, propaedeutic for Nordic walking, lymphoedema and arthralgia, specifically designed for breast cancer survivors. This requires the use of Isa balls, i.e. 6- or 7-cm-diameter foam balls of different densities that can be used either alone or when applied to Nordic walking poles (fig. 1). The series of exercises has the following objectives: to warm up the joints gently, to reduce muscular tension, and to counteract or prevent upper limb lymphoedema. The training scheme started with exercises for hands and wrist joints that were performed using only the Isa balls. These were followed by multi-joint exercises (i.e. wrists, elbows, shoulders) that were carried out using both the Nordic walking poles and the Isa balls applied to them, and by neck exercises that were done using only the Nordic walking poles. The main task during the workout with the Isa balls was to squeeze them gently for the whole duration of each exercise to promote the pumping effects in the upper limbs (fig. 2). After upper limbs and neck exercises, trunk and lower limbs exercises were carried out using the Nordic walking sticks only. Lower limb exercises were performed following a distal to proximal order.

\section{Introduction to Nordic Walking}

Both groups followed the same training scheme: 10 lessons of the Nordic walking technique, 3 times a week, which were supervised by an instructor of the International Nordic Walking Association (INWA). Each lesson followed the same scheme: $15 \mathrm{~min}$ warm-up, $35 \mathrm{~min}$ for the central phase, and $10 \mathrm{~min}$ cool-down. The NW group carried out only the traditional INWA suggested exercises for the warm-up and cool-down. These included whole body mobilisation exercises during the warm-up, and stretching exercises during the cooldown [5]. In contrast, the NW-Isa group performed the Isa method during the warm-up, and both the Isa method and the stretching exercises during the cooldown. During the cool-down, the Isa method did not include lower limbs exercises. The central phase of each lesson was the same in both groups, and included exercises for learning the Nordic walking technique following the INWA
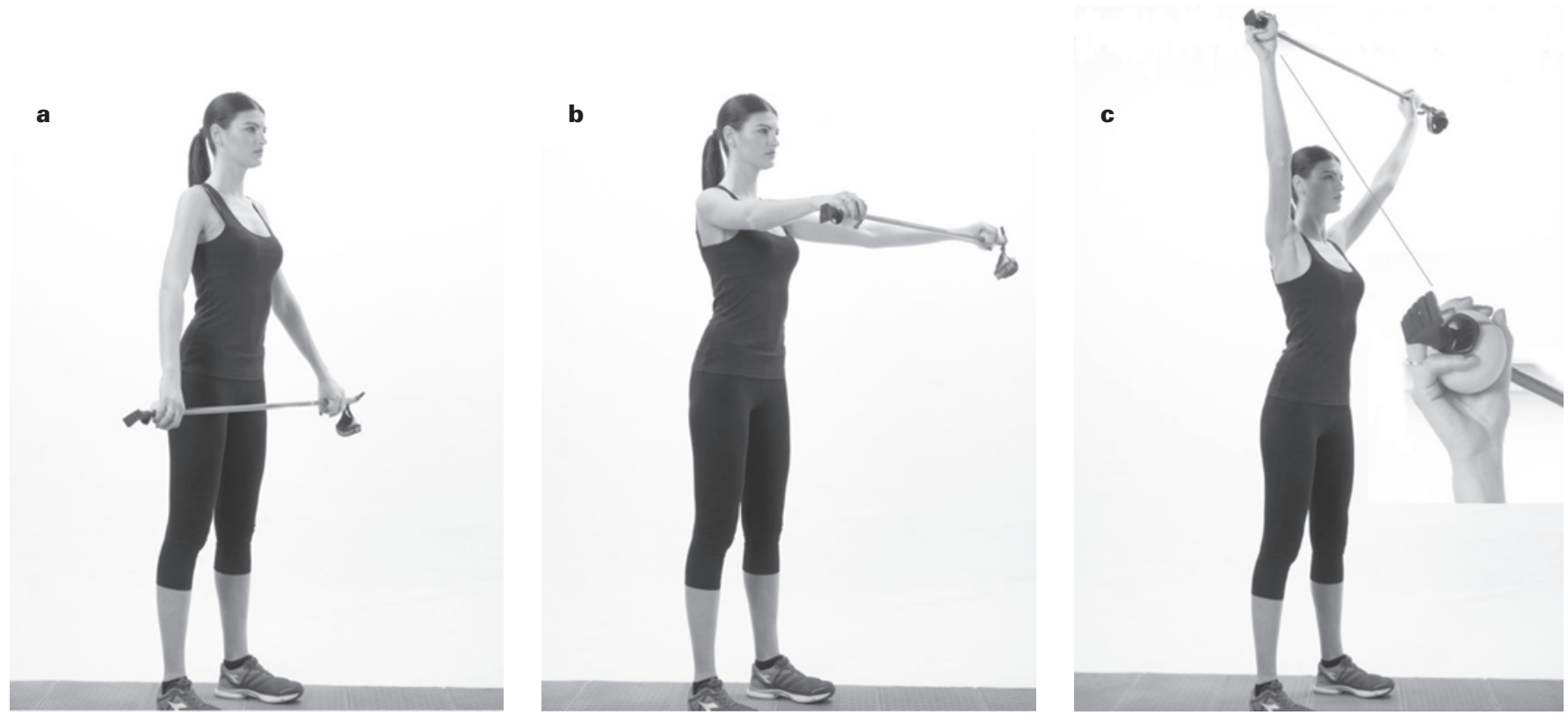

Fig. 2. Example of Isa method exercise. From a to c, correct progression. 
Table 1. Baseline (T0) anthropometric and hydration characteristics of the exercise sub-groups

\begin{tabular}{|c|c|c|c|}
\hline Characteristic & NW group & NW-Isa group & $\mathrm{p}$ \\
\hline Weight, kg & $62.25 \pm 8.87$ & $63.31 \pm 8.13$ & 0.645 \\
\hline Total body water, 1 & $32.98 \pm 2.48$ & $33.20 \pm 4.62$ & 0.645 \\
\hline Extracellular water, 1 & $17.33 \pm 2.11$ & $17.92 \pm 2.09$ & 0.798 \\
\hline $\begin{array}{l}\text { Extracellular water/ } \\
\text { total body water, \% }\end{array}$ & $52.50 \pm 4.27$ & $54.30 \pm 4.18$ & 0.382 \\
\hline \multicolumn{4}{|l|}{ Relaxed arm circ, cm } \\
\hline Homolateral $^{\mathrm{a}}$ & $30.00 \pm 3.46$ & $30.68 \pm 2.36$ & 0.505 \\
\hline Contralateral $^{\mathrm{a}}$ & $30.18 \pm 3.51$ & $30.53 \pm 3.94$ & 0.878 \\
\hline \multicolumn{4}{|l|}{ Max forearm circ, $\mathrm{cm}$} \\
\hline Homolateral $^{\mathrm{a}}$ & $23.71 \pm 1.55$ & $22.95 \pm 2.43$ & 0.959 \\
\hline Contralateral $^{\mathrm{a}}$ & $23.48 \pm 1.58$ & $22.77 \pm 2.55$ & 0.442 \\
\hline \multicolumn{4}{|l|}{ Mid forearm circ, $\mathrm{cm}$} \\
\hline Homolateral $^{\mathrm{a}}$ & $22.16 \pm 1.09$ & $21.80 \pm 2.66$ & 0.878 \\
\hline Contralateral $^{\mathrm{a}}$ & $21.72 \pm 1.19$ & $21.35 \pm 2.86$ & 0.959 \\
\hline \multicolumn{4}{|l|}{ Wrist circ, $\mathrm{cm}$} \\
\hline Homolateral $^{\mathrm{a}}$ & $15.81 \pm 0.61$ & $15.73 \pm 1.17$ & 0.878 \\
\hline Contralateral $^{\mathrm{a}}$ & $15.82 \pm 0.52$ & $15.72 \pm 1.37$ & 0.798 \\
\hline
\end{tabular}

${ }^{a}$ Homolateral/contralateral to the surgical intervention for breast cancer.

$\mathrm{NW}=$ Nordic walking only, NW-Isa $=\mathrm{NW}$ plus the Isa method, $\operatorname{circ}=$ circumference.

Table 2. Analysis of upper limbs measures, according to group membership

\begin{tabular}{lllll}
\hline Group & $\begin{array}{l}\text { Measure, } \\
\text { circumfence }\end{array}$ & $\begin{array}{l}\text { Homolateral } \\
\text { upper limb }, \mathrm{cm}\end{array}$ & $\begin{array}{l}\text { Contralateral } \\
\text { upper limb }\end{array}$ & $\mathrm{p}$ \\
\hline NW & relaxed arm & $30.68 \pm 2.36$ & $30.18 \pm 2.51$ & 0.01 \\
& max forearm & $23.71 \pm 1.55$ & $23.48 \pm 1.58$ & 0.01 \\
& mid forearm & $22.16 \pm 1.09$ & $21.72 \pm 1.19$ & 0.03 \\
& wrist & $15.81 \pm 0.62$ & $15.82 \pm 0.52$ & 0.833 \\
NW-Isa & relaxed arm & $30.53 \pm 3.74$ & $30.00 \pm 3.46$ & 0.02 \\
& max forearm & $22.95 \pm 2.42$ & $22.77 \pm 2.45$ & 0.04 \\
& mid forearm & $21.80 \pm 2.66$ & $21.35 \pm 2.86$ & 0.02 \\
& wrist & $15.73 \pm 1.17$ & $15.72 \pm 1.37$ & 0.915 \\
\hline
\end{tabular}

${ }^{a}$ Homolateral/contralateral upper limb = circumferences taken on the upper limb homolateral/contralateral to the surgical intervention for breast cancer. $\mathrm{NW}=$ Nordic walking only, NW-Isa group = Nordic walking plus Isa method.

scheme [5]. Moving from the first to the last lesson, the central phase shifted its main content from the practice of single exercises to the practice of the com plete Nordic walking technique.

\section{Statistical Analysis}

Statistical analysis was performed using the SAS 9.2 software (SAS Institute Inc., Cary, USA). Although non-parametric tests were performed, due to the small sample size, some data are presented as means \pm standard deviation, to provide a better understanding of the results.

Considering the entire sample, Mann-Whitney tests were used to verify whether the sub-groups differed in their baseline characteristics. The same tests were used for each sub-group, separately, to determine whether the circumferences of the upper limb homolateral to the surgical intervention for breast cancer significantly differed from that of the opposite side. Wilcoxon tests were used to determine (separately for each sub-group) the significantly modified variables at the end of the training period. A p value of $<0.05$ was considered significant.

\section{Results}

Table 1 shows the homogeneity of the NW and the NW-Isa exercise groups. These data demonstrate that neither groups had any significant differences in upper limb circumferences or in total body water and fluid retention. In addition, table 2 shows that for both groups the upper limb homolateral to the surgical intervention for breast cancer had circumferences significantly greater than those of the contralateral upper limb. The only exception was for the wrist.

When Wilcoxon tests were performed following the experimental interventions, the results showed that, while the considered variables were not significantly modified in the NW group, significantly reduced extracellular body water $(\mathrm{p}=0.01)$ and extracellular-to-total body water ratio $(\mathrm{p}=0.01)$ were found in the NW-Isa group. Furthermore, significant reductions were seen in the circumferences of the upper limb homolateral to the surgery for breast cancer: relaxed arm $(\mathrm{p}=0.01)$ and both maximum $(\mathrm{p}=0.01)$ and mid-forearm $(\mathrm{p}=0.01)$ (fig. 3 ). No significant changes were observed for the contralateral limb for the NW-Isa group.

\section{Discussion}

The present study was conducted on a restricted sample, so that in interpreting the data, the concept of 'it might be' instead of 'it is' should be used. However, our data support our starting hypothesis that in the learning phase of Nordic walking, no beneficial effects on lymphoedema in breast cancer survivors are seen, even if our unpublished data confirm that 10 weeks of Nordic walking training are able to reduce lymphoedema. The reason for this may be that, during the learning phase, novice Nordic walkers are not able to adequately generate an effective muscular pump through the coordination of the alternated bimanual open-close cycle. As consequence, the blood and lymphatic upper limb circulation continue to suffer from the effects of gravity through the duration of the lesson. This generates fluid stagnation in the upper limbs, especially in the limb that is homolateral to the surgery and/or radiation therapy.

The Isa method was included here to couple the physiotherapeutic principle (the use of the foam balls against upper limb lymphoedema) with Nordic walking exercises to generate a new system and new progression for the training. Indeed, the combination of Nordic walking with the Isa method appeared to close the gap here. The inclusion of the ISA method exercises for the upper limbs, during both the warm-up and cool-down periods of the sessions for the Nordic walking technique, appeared to reduce both the total body extracellular water and the upper limb circumferences after 10 sessions. Taken together with the physiological concept that 3 weeks are not enough to provide significant fat or muscle mass loss in the absence of a severe negative energy balance, our data indicate that 10 sessions of Nordic walking, combined with the Isa method, can reduce the extracellular fluids of the upper limbs, i.e. reduced lymphoedema. 


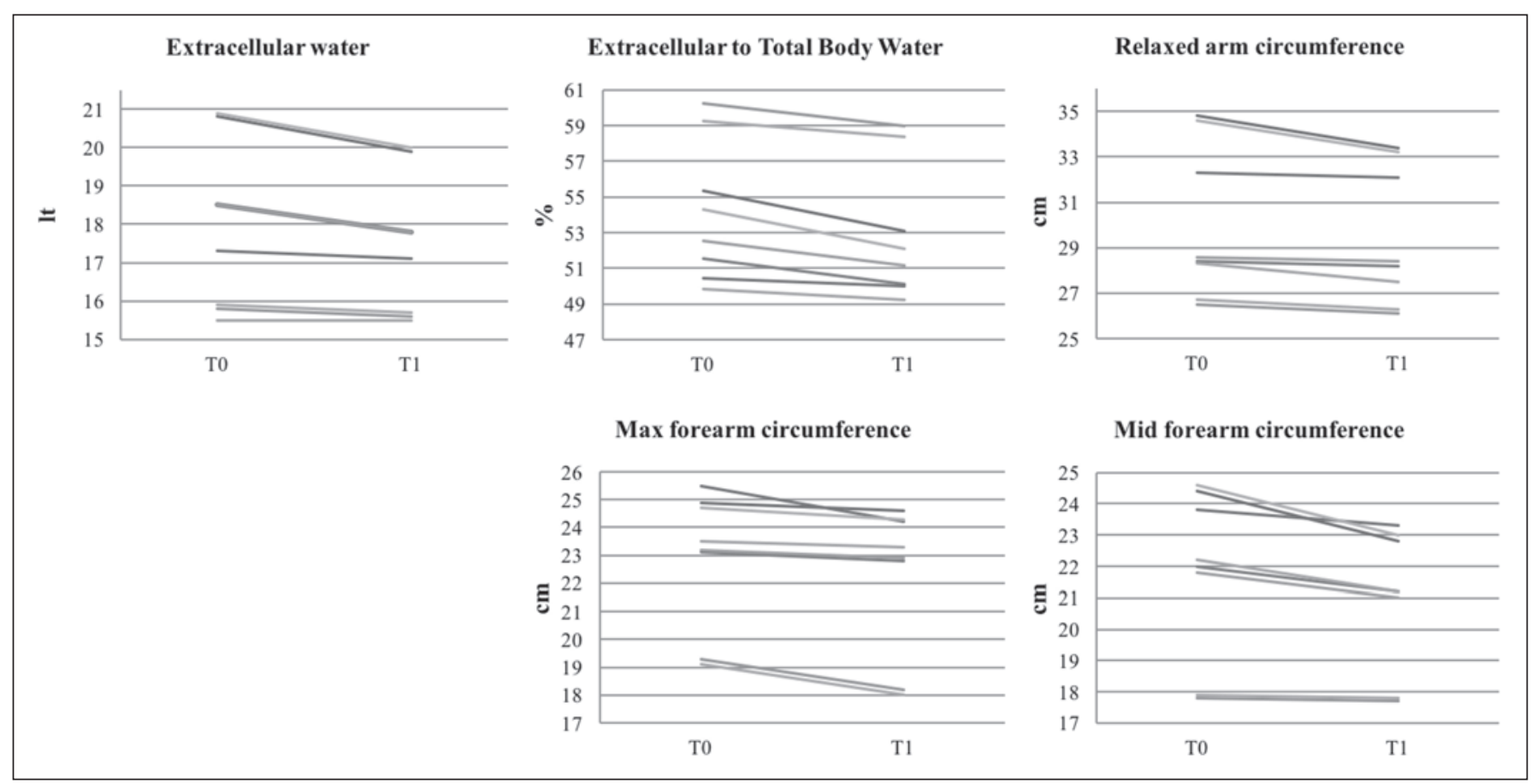

Fig. 3. Significantly modified variables for the Nordic walking plus the Isa method group (NW-Isa) group after 10 sessions (as indicated). T0, baseline; T1, completion of 10 sessions.

This aspect is of a great importance, as it also supports novice Nordic Walkers in their compliance to the exercise, to ensure their participation until the full Nordic walking technique is correctly practiced, and thus before the Nordic walking begins to be effective against the lymphoedema [6,7] and surgery-related kinesiological changes of these breast cancer survivors [8].

In conclusion, a larger study and the use of a more accurate technique to quantify lymphoedema are needed to confirm these results, and to determine whether these results are applicable only to breast cancer survivors with lymphoedema lower than class 2 of the CEAP-L classification. However, even with these limitations, the present study shows that inclusion of the Isa method during the warm-up and cool-down phases of the initial Nordic walking sessions can effectively speed up upper limb lymphoedema improvements for breast cancer survivors.

\section{Online Supplemental Material}

\section{Materials and Methods}

To access the online supplemental material please refer to www.karger. com/?DOI=453599.

\section{Disclosure Statement}

The authors declare that they have no conflicts of interest.

\section{References}

1 Agrawal S: Late effects of cancer treatment in breast cancer survivors. South Asian J Cancer 2014;3:112-115.

2 Wank XS, Woodruff JF: Cancer-related and treatmentrelated fatigue. Gynecol Oncol 2015;136:446-452.

3 Lahart IM, Metsios GS, Nevill AM, Carmichael AM: Physical activity, risk of death and recurrence in breast cancer survivors: A systematic review and meta-analysis of epidemiological studies. Acta Oncol 2015;54:635-654.

4 Shaitelman SF, Cromwell KD, Rasmussen JC, et al.: Recent progress in the treatment and prevention of cancer-related lymphedema. CA Cancer J Clin 2015; 65:55-81.
5 Arrankoski T, da Silva Novaes Coelho DR, da Silva Novaes Coelho TR, et al.: INWA Manual for the Course of Instructor in Nordic walking (in Italian). International Nordic Walking Federation, 2011.

6 Gonzalez Castro C: Nordic walking as a prescribed physical exercise in patients suffering from lymphoedema secondary to breast cancer (in Spanish). Apunts Med Esport 2013;48:97-101.
7 Gonzalez Castro C: Brief overview of the benefits of Nordic walking in the treatment of primary and secondary lymphoedema. Eur J Lymphol Relat Probl 2013;24:28-30.

8 Fischer MJ, Krol-Warmerdam EM, Ranke GM, et al.: Stick together: A Nordic walking group intervention for breast cancer survivors. J Psychosoc Oncol 2015;33: 278-296. 